\title{
Evaluation of the release characteristics of covalently attached or electrostatically bound biocidal polymers utilizing SERS and UV-Vis absorption
}

\author{
G. N. Mathioudakis ${ }^{1,2}$, A. Soto Beobide ${ }^{1 *}$, N. D. Koromilas ${ }^{2}$, J. K. Kallitsis ${ }^{1,2}$, G. Bokias ${ }^{1,2}$, \\ G. A. Voyiatzis ${ }^{1}$
}

${ }^{1}$ FORTH/ICE-HT, P.O. Box 1414, GR-265 04 Rio-Patras, Greece

${ }^{2}$ Department of Chemistry, University of Patras, GR-265 00 Rio-Patras, Greece

Received 18 January 2016; accepted in revised form 30 March 2016

\begin{abstract}
In this work, biocidal polymers with antimicrobial quaternized ammonium groups introduced in the polymer biocidal chains either through covalent attachment or electrostatic interaction have been separately incorporated in a poly (methyl methacrylate) polymer matrix. The objective of present study was to highlight the release characteristics of biocidal polymers, primarily in saline but also in water ethanol solutions, utilizing UV-Vis absorption and Surface Enhanced Raman Scattering (SERS). It is shown that through the combination of UV-Vis and SERS techniques, upon the release process, it is possible the discrimination of the polymeric backbone and the electrostatically bound biocidal species. Moreover, it is found that electrostatically bound and covalently attached biocidal species show different SERS patterns. The long term aim is the development of antimicrobial polymeric materials containing both ionically bound and covalently attached quaternary ammonium thus achieving a dual functionality in a single component polymeric design.
\end{abstract}

Keywords: polymer composites, surface enhanced Raman spectroscopy (SERS), biocidal polymers, release, quaternary ammonium salts

\section{Introduction}

The design of novel environmentally friendly/acceptable biocidal polymeric materials represents a very intensive basic research field, related with modern technological demands. For instance the development of 'clean' surfaces for sensitive sanitary applications or/and alternative antifouling paints to address crucial ecological and environmental problems in marine applications are some of the current challenges we face. The term biocide refers to a substance that mainly acts by inhibiting the growth of microorganisms with harmful consequences not only to human health but also to the quality of several products. The incorporation of antimicrobial substances into polymer matrices gains more and more field in technical hygiene processes as the need for secure 'clean' surfaces for sensitive sanitary applications increases [1, 2]. Moreover, the development of antifouling paints to confront crucial ecological and environmental problems in marine applications is some of the current challenges. An application field of these materials is in the aquaculture sector as well as in marine technology and navigation. In the latter, it is expressed by the marine biofouling, namely the accumulation and the adhesion of organisms, such as barnacle, tube worms and algae, to a surface in contact with water for a long time. Thus, extensive research is conducted in this wide field, concerning between others nets with biocidal properties especially in the aquaculture or/and antifouling paints for sub- 
merged surfaces of the vessels. Polymers with quaternary ammonium or phosphonium salts are widely explored materials with potent antimicrobial activity [3-5]; these cationic biocides target the bacterial membranes. Their antimicrobial activity is based on their ability to disrupt and disintegrate the negatively charged cell membranes. In some cases [6], phosphonium salts show higher activity [7] and higher thermal stability $[8,9]$ however, usually with more demanding chemistry than the quaternary ammonium analogues.

In this specific case, antimicrobial polymeric materials containing quaternary ammonium were developed. They can be classified in two categories depending on the incorporation of the quaternary ammonium salts onto polymers, namely the ionically bound or the covalently attached [10-12]. The polymeric materials of the first category habitually exhibit strong biocidal action based on the release of the active quaternary cation in the vicinity of the aqueous environment through ion exchange mechanism; that allows the dissolution of the polymeric phase and the exploit of the whole biocidal material. However, the biocidal action of the second category is based on the contact of the antimicrobial polymer with the microorganisms, preventing any biocidal release that would burden the marine environment. A note is made of the fact that each category has inherent advantages and disadvantages. Biocidal surfaces bearing immobilized active groups may prevent the formation of viable biofilms; however, they could still be contaminated by remaining dead bacteria that may initiate immune responses and inflammation [13]. From the other side, biocidal surfaces, carrying active agents that could be released, may prevent or reduce the initial attachment of bacteria. Therefore, the combination of both strategies is anticipated to reveal an ideal antibacterial polymeric material; however, till now, few works exhibiting relevant dual antibacterial function have been reviewed $[14,15]$. This study constitutes the preliminary effort to reveal the release characteristics, if any, of polymeric quaternary ammonium biocides of both types in simulated seawater and hopefully highlight the importance of the development of their combination in one antimicrobial polymeric material containing together ionically bound and covalently attached quaternary ammonium groups.
Raman spectroscopy is a widely used non-invasive technique that provides detailed molecular information. Surface Enhanced Raman spectroscopy (SERS) might be proved a superior detection method because it is a very sensitive technique manifested as an enhancement by many orders of magnitude of the intensity of Raman radiation by molecules in the immediate vicinity to nano-rough metal surfaces or nano-structured colloidal clusters of noble metals, such as Ag and Au. SERS constitutes a challenge of applying it to extremely low concentration level analysis. Applying a surface enhanced Raman scattering excitation/collection configuration bearing an oscillating cell and combined with right angle scattering collection geometry we have quite recently introduced a new method to quantitatively monitor the level of active agents at very low concentration range $[16,17]$.

The present work is mainly an attempt to monitor by SERS the release of biocide polymers from polymeric films and compare the release rate obtained with a traditional and consequently most commonly used technique in quantitative analysis of controlled release, such as UV-Vis absorption. In this context, polymeric matrices with the ability of controlled release have been developed; two different biocide polymers (PSSAmC16 and PVBCHAM, see Figure 1) have been incorporated into poly(methyl methacrylate) (PMMA) specimens. These films are new polymeric materials with enhanced (and controlled) antifouling properties, which hold great promise for potential applications in the field of 'clean' surfaces, either for health reasons or as antifouling. The monitoring of the release characteristics of these biocide polymers from the polymeric matrix mainly in saline but also in $95 \%$ ethanol solution has been carried out and compared utilizing both UV-Vis absorption and SERS.
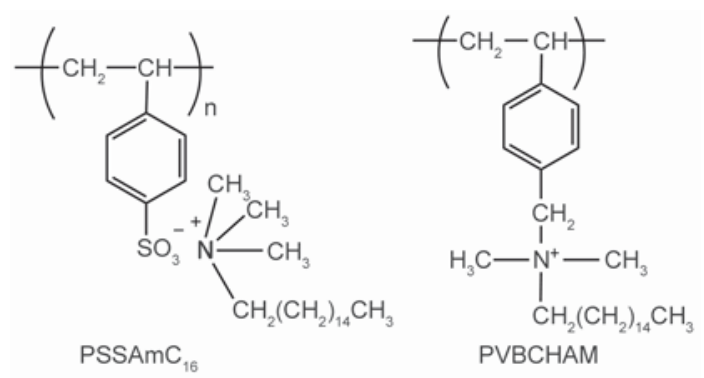

Figure 1. Chemical structures of the polymeric biocides PSSAmC16 (left) and PVBCHAM (right) 


\section{Experimental}

\subsection{Materials}

Poly(methyl methacrylate) (PMMA, 29773) was a product of BDH Chemicals Ltd, Poole England. Hexadecyl trimethyl ammonium bromide (CTAB, H5882) was supplied from Sigma-Aldrich Germany and dimethylacetamide (DMA, O24344) was purchased from Fisher Scientific Europe. The synthesis of poly(styrene hexadecyl trimethyl ammonium sulfonate) (PSSAmC16) and poly(vinyl benzyl dimethyl hexadecyl ammonium chloride) (PVBCHAM) has been described in details elsewhere $[5,10,18,19]$ and their chemical structures are shown in Figure 1. Analytical grade silver nitrate $(>99.99 \%, 209139)$ and sodium citrate tribasic dehydrate $(99.0 \%, \mathrm{~S} 4641)$ for colloid preparation were purchased from SigmaAldrich Germany, too.

\subsection{Preparation of polymer films}

PMMA membranes containing polymeric biocides in a composition 90/10 per weight were prepared by the solution casting technique. The specific biocidal polymer $(0.03 \mathrm{~g})$ and the polymer matrix PMMA $(0.27 \mathrm{~g})$ were dissolved in $5 \mathrm{~mL}$ DMA and heated on a hot plate at $80^{\circ} \mathrm{C}$ under stirring to reach complete dissolution. The polymer mixture solutions were cast on a Pyrex petri plate (diameter $5 \mathrm{~cm}$ ) at $80^{\circ} \mathrm{C}$ for 24 hours, the obtained film was dried in a vacuum oven at $80^{\circ} \mathrm{C}$ for complete solvent removal. The films had a thickness of 100-120 $\mu \mathrm{m}$.

\subsection{Release studies}

For release studies, a piece of film ( $40 \mathrm{mg}$, with dimensions $1.5 \mathrm{~cm} \times 1.5 \mathrm{~cm} \times 0.1 \mathrm{~mm}$ ) was cut and placed in vials filled with $8 \mathrm{~mL}$ of either ethanol $95 \%$ or $\mathrm{NaCl} 0.5 \mathrm{M}$ (seawater simulation) on a Wisd shaking incubator at $50 \mathrm{rpm}$ and $17^{\circ} \mathrm{C}$. The weighted amounts of the specimens were precisely selected so as in the case of total release of biocide agent its concentration into the different solutions to be $0.5 \mathrm{mg} / \mathrm{mL}$ (see UV-Vis absorption calibration curve Figure 2). At several time intervals $2 \mathrm{~mL}$ aliquots were withdrawn and UV-Vis absorption spectra were measured using a double beam Lambda UV/Vis/NIR Spectrometer of Hitachi (U-3000). After the UV-Vis absorption measurement the aliquot was decanted back to the vials.

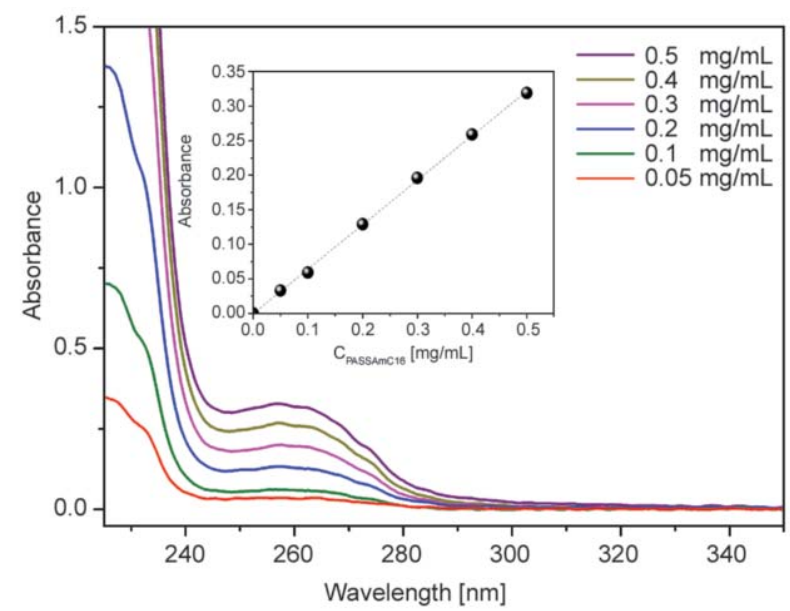

Figure 2. UV-Vis absorption spectra of PSSAmC16/0.5 M $\mathrm{NaCl}$ solutions at various concentrations. Inset: The calibration curve plot absorbance versus concentration extracted from the spectra

The release of biocide polymers from the polymeric matrix was also monitored by Surface Enhanced Raman Scattering (SERS). The colloids used to collect the SERS spectra were prepared according to a modified Lee and Meisel procedure [16, 20]. For these measurements, $100 \mu \mathrm{L}$ aliquots were removed from the vials containing the piece of film immersed in ethanol or $\mathrm{NaCl}$ solution, but this aliquot volume was not returned or replaced. The addition of extra $100 \mu \mathrm{L}$ of the aggregating agent $\mathrm{NaCl}(1 \mathrm{M})$ into $1 \mathrm{~mL}$ of Ag silver colloid gave rise to the more pronounced enhancement. The Raman/SERS spectra were excited with a water-cooled $\mathrm{Ar}^{+}$laser (Spectra physics model 2017, $5 \mathrm{~W}$ all lines) operating at $514.5 \mathrm{~nm}$. An achromatic doublet (f: $145 \mathrm{~mm}$; j: $35 \mathrm{~mm}$ ) was utilized to focus the laser beam into a Pyrex test tube filled with nanocolloidal silver suspensions; the power of the laser beam measured in front of the sample was $40 \mathrm{~mW}$. Raman spectra were collected using the T-64000 spectrometer of Jobin Yvon (ISA-Horiba group). The Raman system was used in the single spectrograph configuration for the collection, analysis and detection of the scattered light. This spectrograph bears a rectangular entrance slit long (and horizontal) in the direction perpendicular to the lateral direction of the separation of light. Therefore, it allows the optimum right angle collection of the collateral scattering volume of a linear-like focusing field of the excitation laser line. For the elastic Rayleigh scattering rejection, an edge filter (LP02-514RU-25, 
Laser 2000 (UK) Ltd.) was used. The Raman photons were dispersed by a 600 -grooves $/ \mathrm{mm}$ blazed holographic diffraction grating and detected by a standard LN2 cooled front illuminated CCD detector. The spectrometer was wavenumber calibrated using the standard Raman band positions of cyclohexane. Reproducible and improved Raman scattering collection measurements were allowed by the use of an 'oscillating cell' in combination with the advantage of utilizing the right angle light scattering collection geometry. A detailed description of this configuration has been reported before [16]. Reference FTRaman spectra were also obtained with NIR excitation at $1064 \mathrm{~nm}$ (via an R510 diode pumped Nd:YAG laser) utilizing a Raman component (FRA-106/S) attached to an Equinox 55 Bruker spectrometer.

\section{Results and discussion}

\subsection{Description of polymeric biocides}

Quaternary ammonium salts are important biocides known to be effective against a broad spectrum of micro-organisms. The antimicrobial efficacy of hexadecyl trimethyl ammonium bromide, CTAB, depends to a great extent on the length of the alkyl chain (hexadecyl chain) [21]. The chemical structures of the biocidal polymers studied here are shown in Figure 1. Hexadecyl trimethyl ammonium cation (AmC16) was incorporated through ion interactions in PSSNa to give as product the homopolymer poly(sodium styrene hexadecyl trimethyl ammonium sulfonate) (PSSAmC16). On the other hand, the quaternized poly(vinyl benzyl dimethyl hexadecylammonium chloride) (PVBCHAM) bears the ammonium group attached covalently on the polymeric chain [10]. The biocidal activity against a broad range of bacteria of the copolymers bearing quaternary ammonium units with potential antibacterial functionalities bound covalently or electrostatically onto the polymeric chain has been studied in parallel [22].

In this work, the ion exchangeable or polymer bound quaternary ammonium groups of PSSAmC16 and PVBCHAM, respectively, are considered, in terms of release ability from the PMMA matrix. Thus, a seawater simulant (aqueous $0.5 \mathrm{M} \mathrm{NaCl}$ solution) or a polar solvent (EtOH 95\%) were used to conduct the study. It should be mentioned that, in the case of PSSAmC16 release in aqueous $0.5 \mathrm{M} \mathrm{NaCl}$ solution, it is unclear whether the AmC16 groups are just ex- changed or the whole PSSAmC16 chain is dissolved. As it will be shown, the combination of SERS and UV-Vis spectroscopy have been proved crucial in this case to discriminate between the AmC16 cations and the poly(styrene sulfonate), PSS, backbone.

\subsection{Calibration curves}

While the solubility of PSSAmC16 in pure water is limited, the polymer is soluble in aqueous $0.5 \mathrm{M} \mathrm{NaCl}$ solution [18]. So, the UV-Vis spectra of PSSAmC16 and CTAB in this seawater simulant were evaluated (Figure 3). The main broad absorption zone of PSSAmC16, bearing bands tentatively located at 256 and $262 \mathrm{~nm}$, is attributed to the benzene ring. On the other hand, the amine based cationic quaternary group in the PSSAmC16, that is CTAB, does not exhibit any significant absorption in the wavelength range under study. Thus, UV-Vis spectroscopy can be used in order to detect the release of PSS backbone of the biocidal polymer in the solution.

Figure 2 depicts the UV-Vis absorption spectra of PSSAmC16 in $0.5 \mathrm{M} \mathrm{NaCl}$ solutions at a concentration range from 0.05 to $0.5 \mathrm{mg} / \mathrm{mL}$ at $0.1 \mathrm{mg} / \mathrm{mL}$ step range. In the inset of Figure 2, the absorbance of the PSSAmC16/ $\mathrm{NaCl}$ solution at $262 \mathrm{~nm}$ is plotted versus concentration, constituting the UV-Vis absorption calibration curve $\left(R^{2}=99.74 \%\right)$ that obeys a linear Beer's law behavior, with a limit of detection $\mathrm{LOD}=28 \mu \mathrm{g} / \mathrm{mL}$ and a limit of quantification, $\mathrm{LOQ}=$ $95 \mu \mathrm{g} / \mathrm{mL}$. Similarly, calibration UV-Vis absorption curves have been performed also for PSSAmC16 and PVBCHAM in $95 \%$ ethanol solution (Figure 4). Figure 4 depicts the UV-vis absorption spectra of $95 \%$

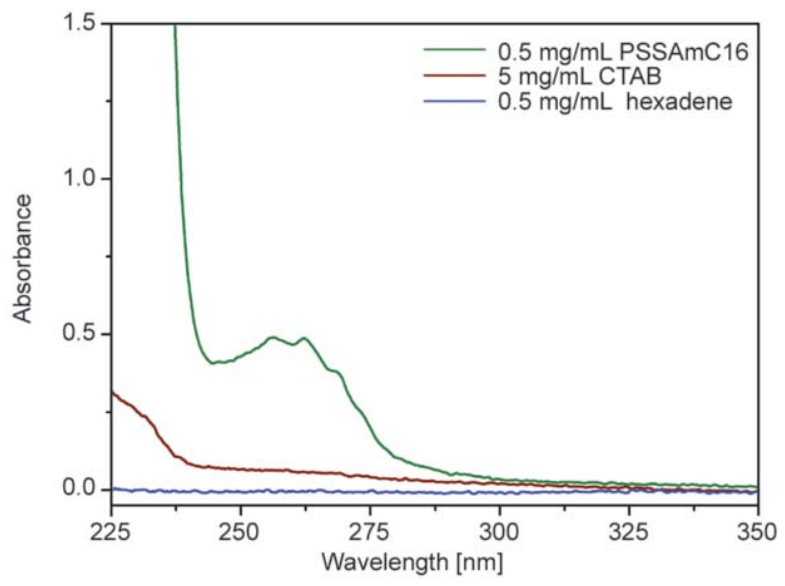

Figure 3. UV-Vis absorption spectra of polymeric biocide PSSAmC16, the quaternary biocide group $\mathrm{CTA}^{+}$ in $0.5 \mathrm{M} \mathrm{NaCl}$ solutions and the aliphatic group Hexadecane in ethanol 

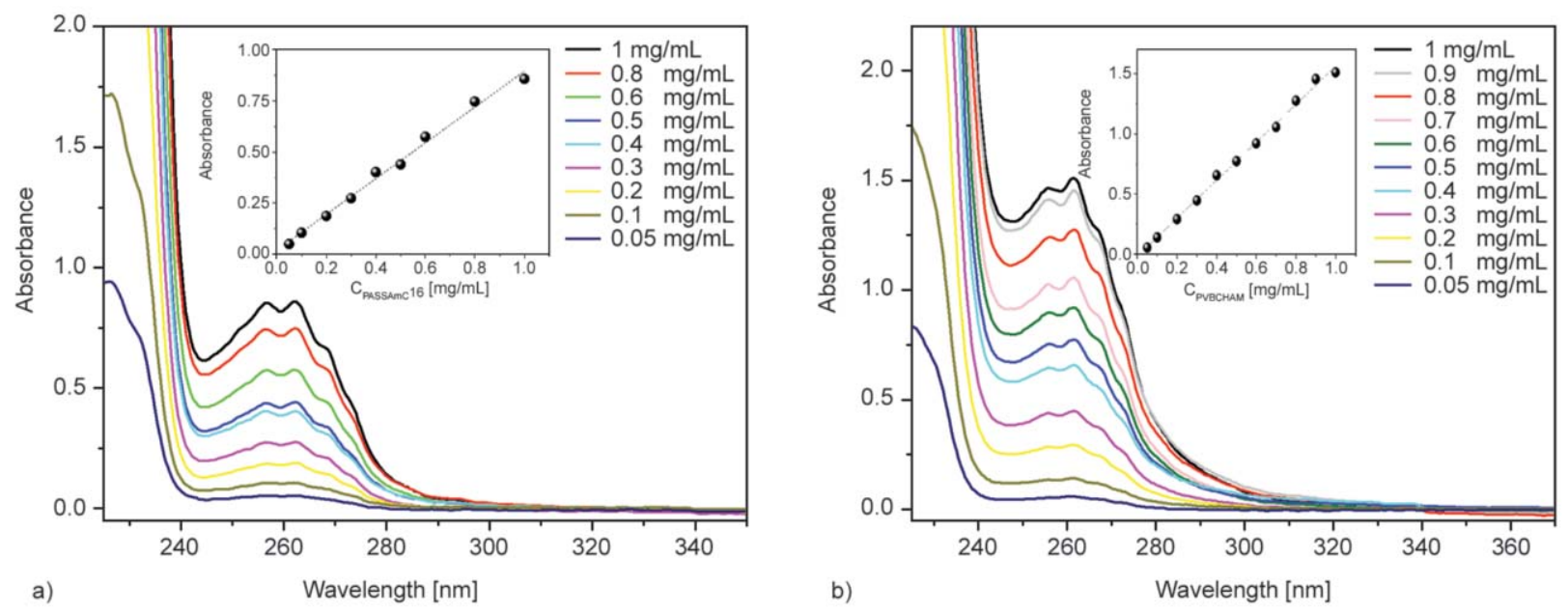

Figure 4. UV-vis absorption spectra of $95 \%$ ethanol solutions of: PSSAmC16 (a) and PVBCHAM (b) at various concentrations. Inset: The calibration curve plots absorbance versus concentration extracted from the spectra

ethanol solutions of PSSAmC16 and PVBCHAM at a concentration range from 0.05 to $1 \mathrm{mg} / \mathrm{mL}$ at $0.1 \mathrm{mg} / \mathrm{mL}$ step range. The main absorption bands $\left(\lambda_{\max }\right)$ of both are located at 256 and $262 \mathrm{~nm}$. In the inset of figures, the absorbance of the polymeric biocides in ethanol solution at $262 \mathrm{~nm}$ is plotted versus concentration constituting the UV-Vis absorption calibration curve that obeys a linear Beer's law behavior. However, since PVBCHAM is not soluble in $\mathrm{NaCl}$, relevant calibration curve could not be obtained.

In Figure 5, the SERS calibration curve of PSSAmC16 in $0.5 \mathrm{M} \mathrm{NaCl}$ solutions is depicted at a concentration range from 10 to $500 \mu \mathrm{g} / \mathrm{mL}$ in the spectral window 1500 to $3200 \mathrm{~cm}^{-1}$. SERS spectra were obtained with a laser power of $40 \mathrm{~mW}$ on sample and a spec-

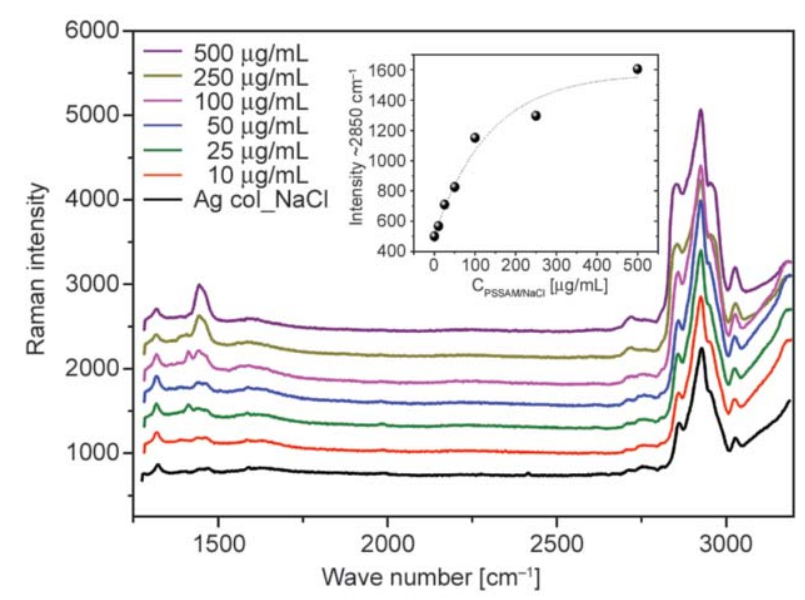

Figure 5. Representative SERS spectra from $100 \mu \mathrm{L}$ of PSSAmC16/0.5 NaCl solutions placed in $1 \mathrm{mLof}$ Ag nano-colloid suspensions activated with $100 \mu \mathrm{L}$ $\mathrm{NaCl} 1 \mathrm{M}$. Inset: calibration curve plot obtained from the intensity values of the peak at $\sim 2850 \mathrm{~cm}^{-1}$ versus concentration tral slit width (SSW) of $\sim 6 \mathrm{~cm}^{-1}$. The main spectral features observed when increasing PSSAmC16 concentration are the peak at $\sim 1460 \mathrm{~cm}^{-1}$ attributed to the $\mathrm{CH}_{2}$ and $\mathrm{CH}_{3}$ bending and the peak at $2848 \mathrm{~cm}^{-1}$ due to $\mathrm{CH}$ stretching in the long methylene chain. For assessment purposes, surface enhanced Raman spectrum of CTAB solution was also obtained. Figure 6 curve c shows the SERS spectrum of a $500 \mu \mathrm{g} / \mathrm{mL}$ $\mathrm{CTAB}$ in $0.5 \mathrm{M} \mathrm{NaCl}$ solution. For comparison, the conventional Raman spectra of the respective solution and of pure solid CTAB are depicted as Figure 6 curve $b$ and curve $a$, correspondingly. On the one side, no scattering spectral features were collected from the particular CTAB solution in the conventional Raman spectrum (Figure 6 curve b). On the other side, comparing the SERS spectrum of CTAB (Figure 6 curve $\mathrm{c}$ ) and the corresponding FT-Raman spectrum

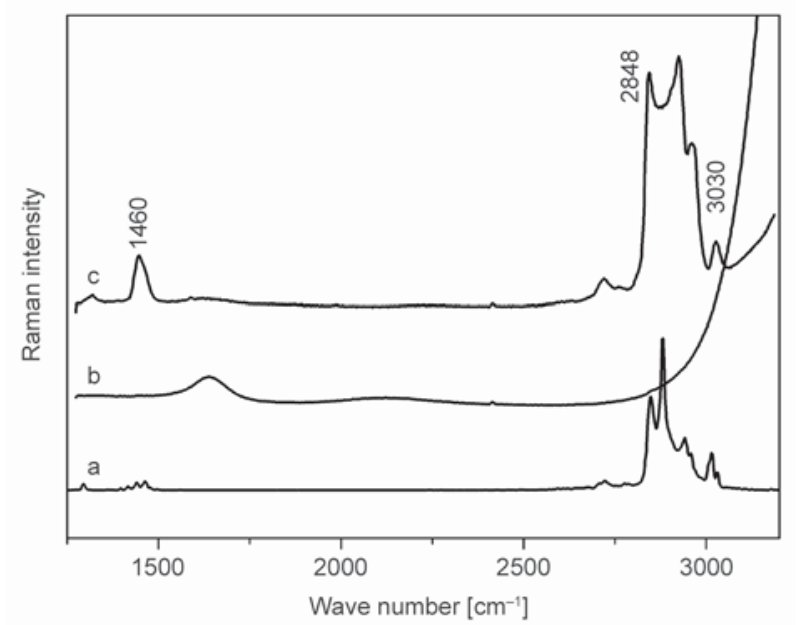

Figure 6. Raman spectra of CTAB: (a) FT-Raman spectrum of pure solid, (b) $0.5 \mathrm{mg} / \mathrm{ml} \mathrm{CTAB}$ solution in $\mathrm{NaCl}$ $0.5 \mathrm{M}$, and (c) SERS spectrum of a $0.5 \mathrm{mg} / \mathrm{mL}$ solution $\mathrm{CTAB}$ in $\mathrm{NaCl}$ 
obtained from the solid (Figure 6 curve a), similarities are found. However, there is an unusually large intensity enhancement of the band at $\sim 1460 \mathrm{~cm}^{-1}$ $\left(\mathrm{CH}_{2}\right.$ and $\mathrm{CH}_{3}$ bending) and the predominance of the band at $2848 \mathrm{~cm}^{-1}(\mathrm{CH}-$ stretching region). The band at $3030 \mathrm{~cm}^{-1}$ is due to the head group $\left(\mathrm{CH}_{3}\right)_{3} \mathrm{~N}^{+}$stretch deformations. Dendramis et al. [23] have performed a detailed SERS study of CTAB and they concluded that it is not unreasonable to assign the $\sim 1460 \mathrm{~cm}^{-1}$ band primarily to deformation of methyl groups attached to the quaternary nitrogen and suggested that the CTAB chain has most $\mathrm{C}-\mathrm{C}$ bonds nearly parallel to the surface enhancing the Raman signal, while the head group is in close proximity to the surface with the three methyl groups positioned symmetrically over it. We would like to point out that we also attempted to obtain SERS spectrum of hexadecane (long chain methylene in $\mathrm{CTAB}, \mathrm{CH}_{3}\left(\mathrm{CH}_{2}\right){ }_{14} \mathrm{CH}_{3}$ ) dissolved in ethanol, since it is practically insoluble in water, but no vibrational spectrum was collected. Anyway, this certainly suggests that positively charged analyte, in the case of CTAB the head group $\left(\mathrm{CH}_{3}\right)_{3} \mathrm{~N}^{+}$, is required for SERS when using citratereduced silver nanoparticles colloid (negative surface charge).

Comparing the SERS spectra in Figure 5 with the one in Figure 6 curve c, it is evident that the surface enhanced spectrum of PSSAmC16 coincides with the spectrum of CTAB; no additional peaks due to the poly(styrene sulfonate) are observable, and this is corroborated by the absence of spectral features due to the ring in the SERS spectra of PSSNa, which is similar to that of the silver colloid activated with $100 \mu \mathrm{L} \mathrm{NaCl}$ (Figure 7).

The usefulness of SERS as an accurate mean of determining the release of quaternary biocide group (AmC16) from the polymer PSSAmC16 can be use in parallel with the ability of UV-Vis absorption technique that allows the characterization of the poly (styrene sulfonate) group (PSS). As we have already established (Figure 3), the quaternary AmC16 group of PSSAmC16 does not exhibit any significant absorption band in the UV-Vis wavelength under study. This confirms that the absorption band obtained when measuring the UV-Vis spectra of PSSAmC16 solutions are due to the styrene sulfonate groups of the PSS chain. At the same time, the PSS chain does not present any spectral feature when trying to obtain

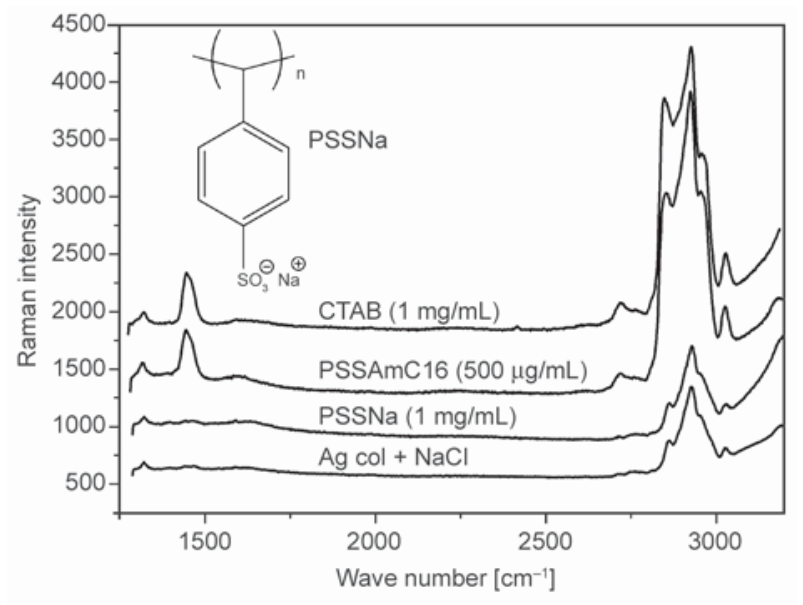

Figure 7. SERS spectra of CTAB, PSSAmC16 and PSSNa in $\mathrm{NaCl}$ solutions. $\mathrm{Ag}$ colloid is given for reference.

the SERS spectrum of a PSSNa solution (Figure 7), as verified by the complete absence of Raman signal from a $1 \mathrm{mg} / \mathrm{mL}$ PSSNa/ $\mathrm{NaCl}$ solution when added to Ag colloid. The spectral features in the SERS spectra of PSSAmC16 are due to the CTAB group, since the SERS spectra of PSSNa is similar to that of the silver colloid activated with $100 \mu \mathrm{L} \mathrm{NaCl}$. Namely, the strong similarity of the surface enhanced spectrum of the PSSAmC16/NaCl solution with the CTAB/ $\mathrm{NaCl}$ solution confirms that the spectral features are due to the cationic quaternary biocide group (AmC16).

The second biocidal polymer under study is the quaternized poly (vinyl benzyl dimethyl hexadecylammonium chloride) (PVBCHAM) that bears the quaternary nitrogen attached covalently on the polymeric chain. This polymer is not soluble in $\mathrm{NaCl}$ as there is no possibility of ion exchange since the biocidal group is covalently attached to the chain. That is, the release studies of PVBCHAM from the PMMA polymeric matrix were performed in $95 \%$ ethanol solutions. Therefore, calibration curves of PVBCHAM in $95 \%$ ethanol solutions were elaborated using UVVis (Figure 4b) and SERS (Figure 8) measurements. Absorption spectra present the characteristic bands of the aromatic ring at 256 and $262 \mathrm{~nm}$ as in the case of PSSAmC16. The corresponding SERS spectral features of PVBCHAM (Figure 8) are not identical to those of AmC16. The $2848 \mathrm{~cm}^{-1}$ Raman peak attributed to $\mathrm{CH}-$ stretching and the Raman peak at $3030 \mathrm{~cm}^{-1}$ due to the head group $\left(\mathrm{CH}_{3}\right)_{3} \mathrm{~N}^{+}$stretch deformations in $\mathrm{AmC} 16$ are not the predominant ones 


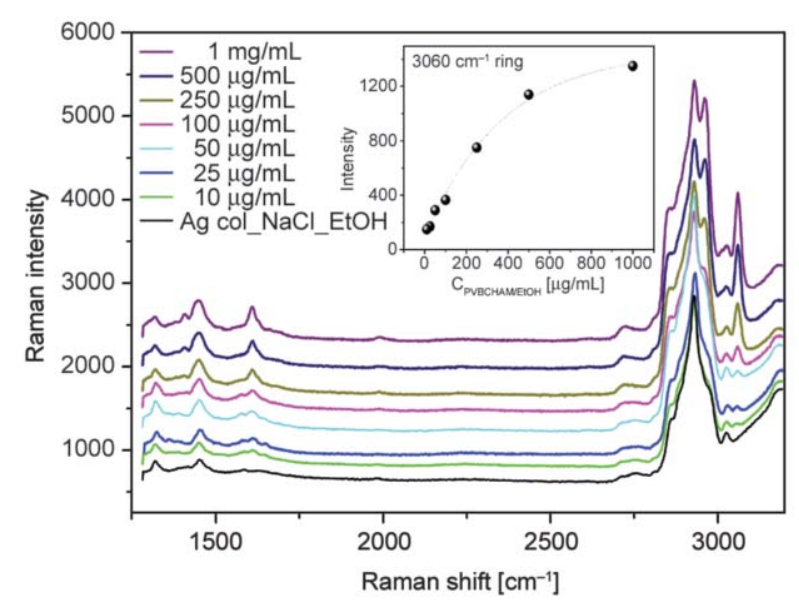

Figure 8. Representative SERS spectra from $100 \mu \mathrm{L}$ of PVBCHAM/95\%ethanol solutions placed in $1 \mathrm{~mL}$ of Ag nano-colloid suspensions activated with $100 \mu \mathrm{L} \mathrm{NaCl} 1 \mathrm{M}$. Inset: calibration curve plot obtained from the intensity values of the peak at $\sim 3060 \mathrm{~cm}^{-1}$ versus concentration

as in the case of PSSAmC16, but in the case of PVBCHAM new additional SERS features are generated at $\sim 1613$ and $3060 \mathrm{~cm}^{-1}$. These new SERS peaks can be respectively assigned to the $1-4$ disubstituted benzene ring (peak at $\sim 1613 \mathrm{~cm}^{-1}$ ) and the aromatic $\mathrm{CH}$ stretching mode (peak at $3060 \mathrm{~cm}^{-1}$ ). On the other hand, we already know that in the case of PVBCHAM the biocide is covalently attached so there is not free quaternary species in the solution. As a consequence of electrostatic interactions, the polycation PVBCHAM most probably approaches close or even is attached to the negatively surface charged citrate-reduced silver nanoparticles and enhancement of the Raman peaks assigned to the aromatic ring occurs. This is an important issue, revealing the CTAB type of moieties as potential carriers stimulating SERS activity to attached presumably SERS inactive functional groups.

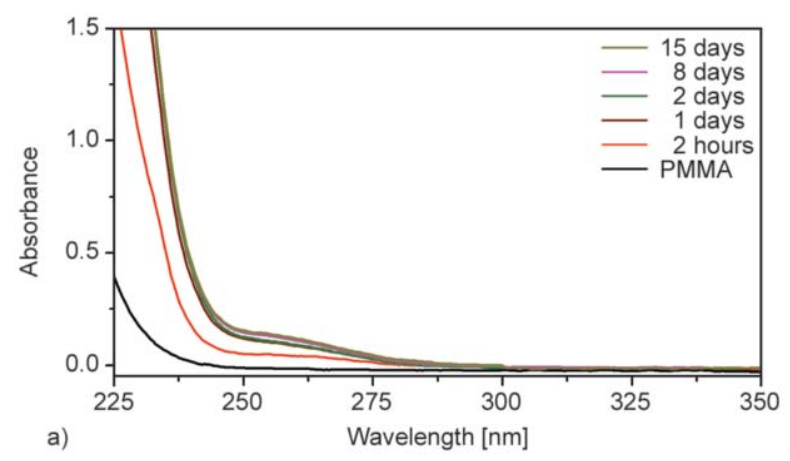

The corresponding SERS spectra of the biocidal polymers, PSSAmC16 and PVBCHAM, despite their similar structure, display some different spectral features, due to their own way of 'adsorption - interaction' at the colloid surface revealing distinct SERS features that enable discrimination between them. Based on these considerations/facts, it is then reasonable to assume the possibility of carrying out the main objective of present work highlighting the release characteristics of quaternary ammonium-based biocidal polymers, mainly in saline, but also in water ethanol solutions, utilizing UV-Vis absorption and SERS.

\subsection{Release measurements}

The release characteristics of the quaternary ammonium biocide group, either ionically attached or covalently bound, have been explored by a systematic study, via both UV-Vis absorption and SERS, performed for PMMA films containing PSSAmC16 or PVBCHAM and immersed into either $0.5 \mathrm{M} \mathrm{NaCl}$ (seawater simulate) or $95 \%$ ethanol solutions (accelerated release study) for a period of time extended up to a few weeks.

\subsubsection{Immersion of $\sim 40 \mathrm{mg}$ of PMMA films doped with biocide polymer into $8 \mathrm{~mL}$ of $0.5 \mathrm{M} \mathrm{NaCl}$ solution}

In Figure 9, we monitor both by UV-Vis (Figure 9a) and SERS (Figure 9b) any eventual release after the immersion of a PMMA/PSSAmC16 (90/10) film in $0.5 \mathrm{M} \mathrm{NaCl}$ solution for a period of time from a few hours up to 15 days. In the UV-Vis spectra, the absorption values are essentially close to the baseline with the maximum value at 0.10 after 15 days of immersion. According to the calibration curve of Figure 2, this could correspond to a concentration of

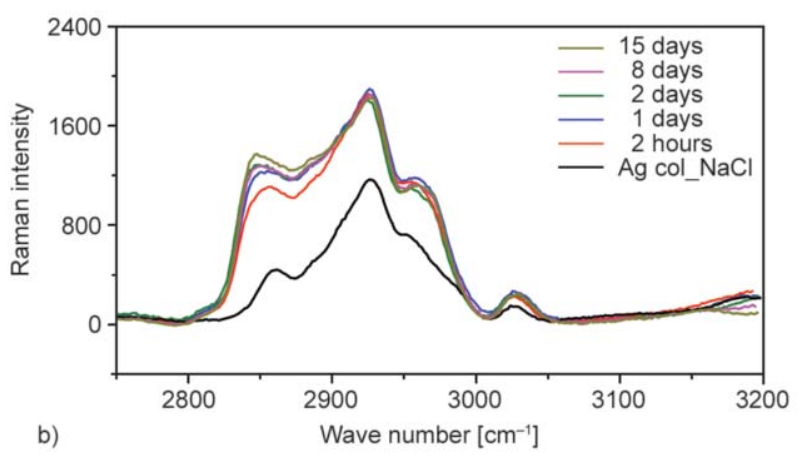

Figure 9. (a) UV-Vis absorbance spectra of the $0.5 \mathrm{M} \mathrm{NaCl}$ solution after the immersion of PMMA/PSSAmC16 (90/10) film for a period of time up to 15 days. (b) SERS spectra 
PSS of $\sim 150 \mu \mathrm{g} / \mathrm{mL}$. The absorption spectrum of a $0.5 \mathrm{M} \mathrm{NaCl}$ solution where a pure PMMA film (without loaded biocide polymer) was immersed is also shown for comparison; it does not exhibit any absorption at $\sim 262 \mathrm{~nm}$ regardless of the time of immersion. In the SERS spectra depicted on the Figure 9b, we can observe the corresponding vibrational spectroscopic features of PSSAmC16, in this case via the $\mathrm{CTAB}$ molecular fingerprint. There is an early release of AmC16 from the biocide doped PMMA film in the saline solution in the 2 first hours of immersion. Afterwards, during the next couple of weeks, it converges towards a plateau that corresponds to a concentration of AmC16 of $\sim 200 \mu \mathrm{g} / \mathrm{mL}$, according to the calibration curve in Figure 5. Therefore, the concentration of both ionic components of PSSAmC16 released, PSS and $\mathrm{AmC1} 16$, in $8 \mathrm{~mL}$ of a $0.5 \mathrm{M} \mathrm{NaCl}$ solution after the immersion for 15 days of a $\sim 40 \mathrm{mg}$ PMMA/PSSAmC16 (90/10) film was effectively determined by the combination of UV-Vis and SERS. From these results, the overall quantity of the released material, namely the sum of the masses of its components found in solution, is determined at $2.8 \mathrm{mg}$. This quantity is $\sim 70 \%$ of the PSSAmC16 content (4 mg) of the PMMA film, in a very good agreement with the results found through Total Organic Carbon and Total Nitrogen determination for the release of PSSAmC16 for similar release studies [18]. The projection of the concentration to the moles of PSSAmC16 released after a few weeks of immersion reveals a number of $0.80 \cdot 10^{-6}$ moles of styrene sulfonate units of PSS detected by the UV-Vis measurements vis-àvis to the number of $0.70 \cdot 10^{-6}$ moles of $\mathrm{AmC} 16$ cations probed by SERS measurements.

Other PMMA film loaded with the covalently attached quaternary ammonium group, PVBCHAM, was also subjected to the same release conditions in $0.5 \mathrm{M} \mathrm{NaCl}$ solution. The UV-Vis measurements of aliquots measured at similar time intervals gave rise to very low absorption values even after the long period of time (15 days), coinciding with the absorption profile of the reference PMMA sample. The same applies for the corresponding SERS measurements; no vibrational spectral features were observed. That is, there is no release of PVBCHAM from the $10 \mathrm{wt} \%$ PVBCHAM incorporated PMMA film when immersed in $\mathrm{NaCl} 0.5 \mathrm{M}$.

\subsubsection{Immersion of $\sim 40 \mathrm{mg}$ of PMMA films doped with biocide polymers into $8 \mathrm{~mL}$ of $\mathbf{9 5 \%}$ ethanol solution}

This time, PMMA/biocide samples were immersed in $95 \%$ ethanol solutions and the release of the biocide group was again studied by both UV-Vis and SERS. Figures 10 and 11 show the results for samples PMMA/PSSAmC16 (90/10) and PMMA/ PVBCHAM (90/10), respectively, probed by both techniques. In the UV-Vis measurements shown as Figures 10a and 11a, the absorption spectrum of a 95\% ethanol solution where a pure PMMA film (without loaded biocide polymer) was immersed is also shown as control in the two extreme time intervals of the release process adopted. There is certain absorption indicating that an amount of the polymer is present in the ethanol solution and increases with time due to the partial solubility of PMMA in 95\% ethanol. The absorption is due to the carbonyl chromophore $(\mathrm{C}=\mathrm{O})$ of PMMA $\left(n \rightarrow \pi^{*} 270 \mathrm{~nm}\right.$, and $\left.\pi \rightarrow \pi^{*} 170-200 \mathrm{~nm}\right)$. Therefore, in the biocide doped PMMA films immersed in ethanol solutions, there is an increasing contribution of the $\sim 262-270 \mathrm{~nm}$ absorption zone due to the partial dissolution of the poly-

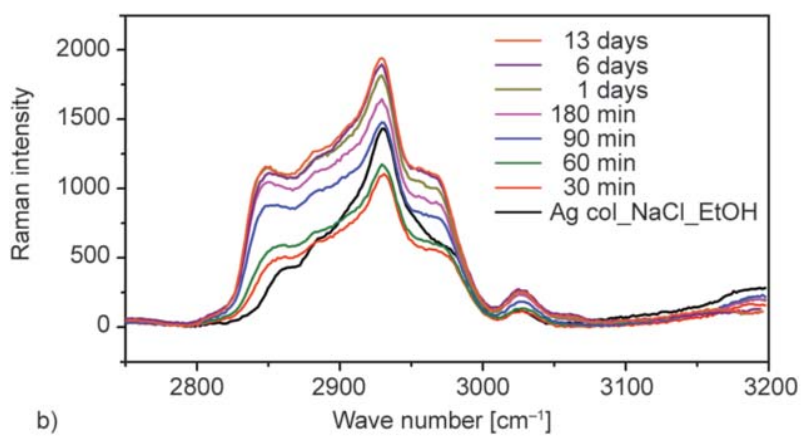

a)

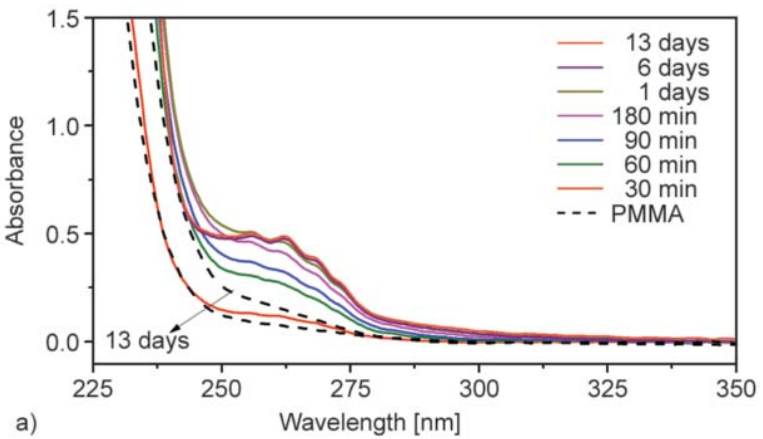

Figure 10. (a) UV-Vis absorbance spectra of the $95 \%$ ethanol solution after the immersion of PMMA/PSSAMC16 (90/10) film for a period of time up to 13 days. The UV-Vis spectra of an ethanol solution where a PMMA film was immersed (as blank) for a few minutes and for a period of 13 days are depicted with dash line. (b) SERS spectra 

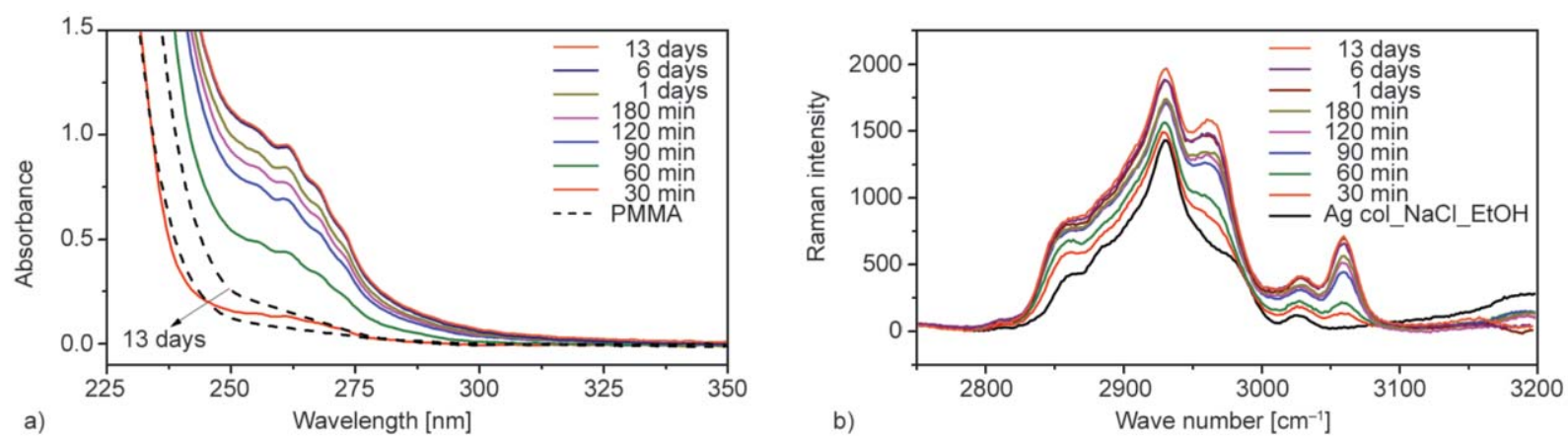

Figure 11. (a) UV-Vis absorbance spectra of the $95 \%$ ethanol solution after the immersion of PMMA/PVBCHAM (90/10) film for a period of time up to 13 days. The UV-Vis spectra of an ethanol solution where a PMMA film was immersed (as blank) for a few minutes and for a period of 13 days are depicted with dash line. (b) SERS spectra

mer matrix, as well. Taking into account the above, we may have a look at the release of either PSSAmC16 (Figure 10) or PVBCHAM (Figure 11) in 95\% ethanol solution. It is clearly seen that at the beginning of the release process, in the first 2-3 hours, there is a 'burst' effect with elevated absorption values at $\sim 262 \mathrm{~nm}$. The absorption values in the case of PVBCHAM biocide polymer encapsulated in PMMA polymer matrix are higher than the corresponding bearing the PSSAmC16 one. This is in agreement with the higher absorption values of PVBCHAM with respect to PSSAmC16 in 95\% ethanol calibration curves given in Figure 4. This burst effect can be explained by the swelling of PMMA polymer matrix in ethanol solutions [24], allowing the biocide polymer to be leached out of the polymer matrix. In the corresponding SERS experiments, shown in Figures $10 \mathrm{~b}$ and $11 \mathrm{~b}$, a similar early release is observed for both PSSAmC16 and PVBCHAM biocide doped PMMA films immersed in $95 \%$ ethanol solutions.

In order to investigate the morphology and quality of mixing between the biostatic polymer and the polymeric matrix, cross section SEM images of biocidefree PMMA polymer films as well as after PSSAmC16 or PVBCHAM biocides incorporation before and after the immersion in $\mathrm{NaCl} 0.5 \mathrm{M}$ and ethanol $95 \%$ aqueous solutions are shown in Figure 12. The fractured specimens were examined utilizing a SUPRA 35VP Zeiss scanning electron microscope. In Figures $12 b$ and $12 c$, the biocides are shown to be dispersed in the PMMA matrix bearing micro droplet morphology, as compared to Figure 12a. After the immersion of the PMMA/biocide films in $\mathrm{NaCl} 0.5 \mathrm{M}$ solution only the electrostatically bound PSSAmC16 has been released; as a consequence, craters were observed on the PMMA/PSSAmC16 films (Figure 12e) to the locations occupied by the biocide before. Conversely, in Figure $12 \mathrm{f}$ the SEM picture presents similar morphology to the corresponding of the control film (Figure 12c) since the covalently attached PCVBHAM is not released from the polymer matrix after the immersion in $\mathrm{NaCl}$. Finally, when PMMA/ biocide polymer films were immersed in ethanol solutions the release/dissolution of both biocides, PSSAmC16 and PVBCHAM, generates hollows in the polymeric matrix indicating a burst type release aided by the swelling or/and even the partial dissolution of the PMMA matrix (Figures 12g, h and i).

\section{Conclusions}

Surface enhanced Raman scattering allows the detection and more over the discrimination between similar biocide polymers bearing a quaternary ammonium group (hexadecyl trimethyl ammonium unit) introduced in the polymer chain through electrostatic or covalent interaction. In the case of electrostatic interaction (PSSAmC16), the bands corresponding to the head group vibration, $\left(\mathrm{CH}_{3}\right)_{3} \mathrm{~N}^{+}$, appear to be preferentially enhanced, while in the case of covalent interaction (PVBCHAM), the bands enhanced are the ones belonging to the aromatic ring. This can be explained by the way the biocide polymers approximate the negatively charge citrate reduced silver colloid used for the SERS measurements. For PSSAmC16, there is ion exchange in $\mathrm{NaCl}$ solutions and it is the AmC16 group the one that interacts with the colloid. On the other hand, the polycation PVBCHAM interacts electrostatically with the citrate reduced silver colloid, resulting in the close proximity of the aromatic ring to the silver colloid and enhancing thus the Raman bands corresponding to the ring. 


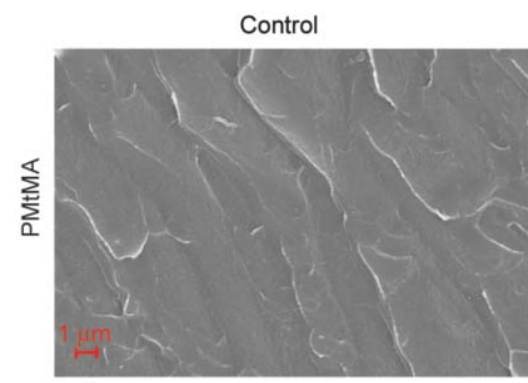

a)

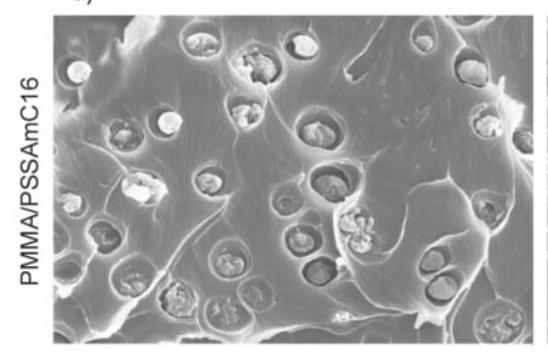

b)

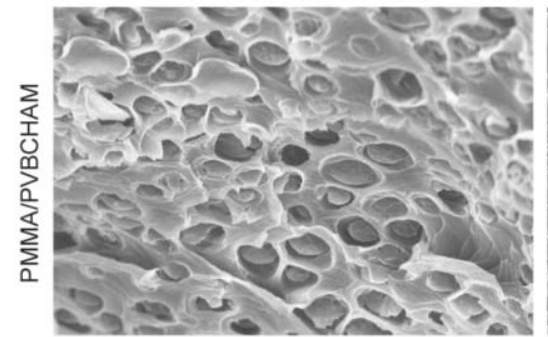

c)
$\mathrm{NaCl} 0.5 \mathrm{M}$

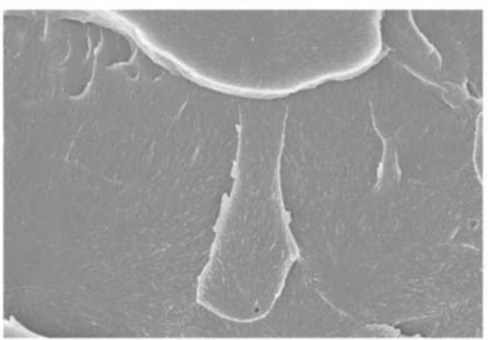

d)

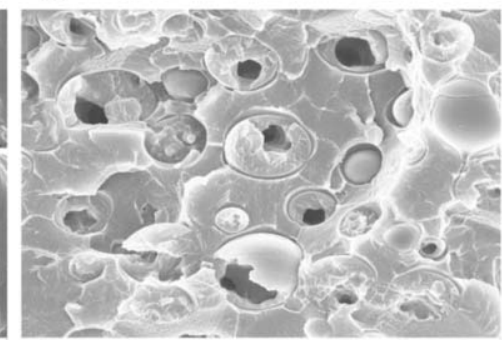

e)

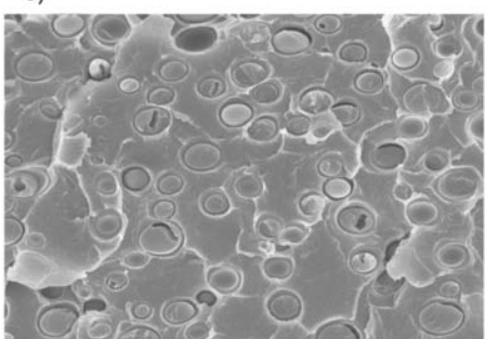

f)

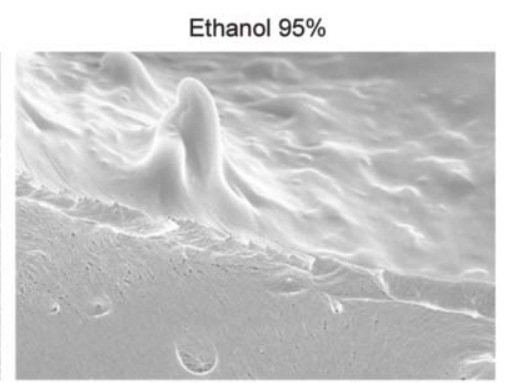

g)

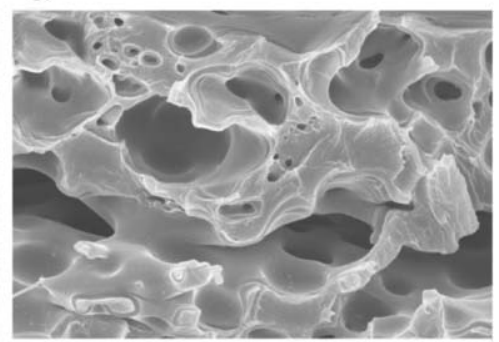

h)

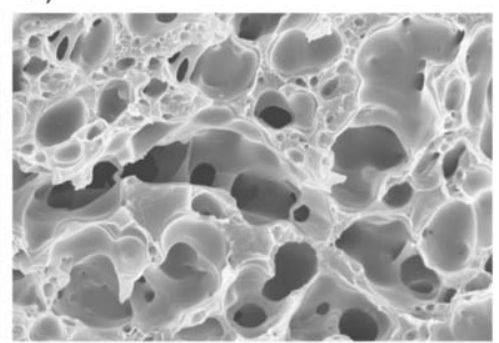

i)

Figure 12. Film morphologies by SEM. Control films: PMMA (a), PMMA/PSSAmC16 90/10 (b) and PMMA/PVBCHAM 90/10 (c). Films immersed in 0.5 M NaCL: PMMA (d), PMMA/PSSAmC16 90/10 (e) and PMMA/PVBCHAM 90/10 (f). Films immersed in 95\% ethanol: PMMA (g), PMMA/PSSAmC16 90/10 (h) and PMMA/PVBCHAM 90/10 (i).

From a practical point of view, in this work SERS and UV-Vis measurements were applied to study the release of biocidal polymers from a polymer matrix. When the polymer matrix bearing the biocide polymer is immersed in seawater simulation solution, the release of PSSAmC16 (the biocidal quaternized ammonium group is ionically attached to the polymer chain) can be detected by both methods; UV-Vis absorption is able to detect the polymer chain PSS and SERS measurements can identify the AmC16 group. This is due to dissociation of the PSSAmC16 in the $\mathrm{NaCl}$ solution through ion exchange in which PSSAmC16 is soluble. These kind of antimicrobial polymers are known as biocide-releasing polymers. However, in the case of PVCHAM (the biocidal quaternized ammonium group is attached to the polymer chain by covalent bond) there is not release to the $\mathrm{NaCl}$ solution due to insolubility. When the release study is performed in ethanol solutions, the biocidal polymer is expelled out the polymer matrix and detected by both, absorption and SERS measurements.
Overall, UV-Vis and SERS methodologies constitute a powerful combination, enabling us to discriminate the polymeric PSS backbone and the electrostatically bound AmC16 species, as well as to discriminate between electrostatically bound and covalently attached PVBCHAM species. This is of utmost importance for the development of antimicrobial polymeric materials containing both ionically bound and covalently attached biocidal quaternary ammonium units, achieving thus a dual functionality in a single component polymeric design.

\section{Acknowledgements}

This research has been co-financed by the European Union (European Social Fund - ESF) and Greek national funds through the Operational Program 'Education and Lifelong Learning' of the National Strategic Reference Framework (NSRF) - Research Funding Program: THALES. Investing in knowledge society through the European Social Fund. Project title: 'Development of Novel Functional Copolymers and Surfaces with Permanent and/or Controlled released biocidal species' (MIS: 379523). 


\section{References}

[1] Muñoz-Bonilla A., Fernandez-Garcia M.: Polymeric materials with antimicrobial activity. Progress in Polymer Science, 37, 281-339 (2012). DOI: $10.1016 /$ j.progpolymsci.2011.08.005

[2] Muñoz-Bonilla A., Fernandez-Garcia M.: The roadmap of antimicrobial polymeric materials in macromolecular nanotechnology. European Polymer Journal, 65, 46-62 (2015).

DOI: 10.1016/j.eurpolymj.2015.01.030

[3] Gilbert P., Moore L. E.: Cationic antiseptics: Diversity of action under a common epithet. Journal of Applied Microbiology, 99, 703-715 (2005).

DOI: 10.1111/j.1365-2672.2005.02664.x

[4] Saif M. J., Anwar J., Munawar M. A.: A novel application of quaternary ammonium compounds as antibacterial hybrid coating on glass surfaces. Langmuir, 25, 377-379 (2009).

DOI: $10.1021 / 1 \mathrm{a} 802878 \mathrm{p}$

[5] Oikonomou E. K., Iatridi Z., Moschakou M., Damigos P., Bokias G., Kallitsis J. K.: Development of $\mathrm{Cu}_{2}{ }^{+}-$ and/or phosphonium-based polymeric biocidal materials and their potential application in antifouling paints. Progress in Organic Coatings, 75, 190-199 (2012). DOI: 10.1016/j.porgcoat.2012.04.019

[6] Xue Y., Xiao H., Zhang Y.: Antimicrobial polymeric materials with quaternary ammonium and phosphonium salts. International Journal of Molecular Sciences, 16, 3626-3655 (2015).

DOI: $10.3390 /$ ijms 16023626

[7] Kanazawa A., Ikeda T., Endo T.: Polymeric phosphonium salts as a novel class of cationic biocides. II. Effects of counter anion and molecular weight on antibacterial activity of polymeric phosphonium salts. Journal of Polymer Science Part A: Polymer Chemistry, 31, 1441-1447 (1993).

DOI: 10.1002/pola.1993.080310611

[8] Wolff M. O., Alexander K. M., Belder G.: Uses of quaternary phosphonium compounds in phase transfer catalysis. Chimica Oggi, 18, 29-32 (2000).

[9] Bradaric C. J., Downard A., Kennedy C., Robertson A. J., Zhou Y.: Industrial preparation of phosphonium ionic liquids. Green Chemistry, 5, 143-152 (2003).

DOI: 10.1039/B209734F

[10] Koromilas N. D., Lainioti G. C., Oikonomou E. K., Bokias G., Kallitsis J. K.: Synthesis and self-association in dilute aqueous solution of hydrophobically modified polycations and polyampholytes based on 4-vinylbenzyl chloride. European Polymer Journal, 54, 39-51 (2015).

DOI: 10.1016/j.eurpolymj.2014.02.009
[11] Guo A., Wang F., Lin W., Xu X., Tang T., Shen Y., Guo S.: Evaluation of antibacterial activity of $\mathrm{N}$-phosphonium chitosan as a novel polymeric antibacterial agent. International Journal of Biological Macromolecules, 67, 163-171 (2014). DOI: $10.1016 /$ j.ijbiomac.2014.03.024

[12] Kenawy E-R., Abdel-Hay F. I., El-Shanshoury A. E. R. R., El Newehy M. H.: Biologically active polymers. V. Synthesis and antimicrobial activity of modified poly (glycidyl methacrylate-co-2-hydroxyethyl methacrylate) derivatives with quaternary ammonium and phosphonium salts. Journal of Polymer Science Part A: Polymer Chemistry, 40, 2384-2393 (2002).

DOI: $10.1002 /$ pola.10325

[13] Costerton J. W., Stewart P. S., Greenberg E. P.: Bacterial biofilms: A common cause of persistent infections. Science, 284, 1318-1322 (1999). DOI: $10.1126 /$ science. 284.5418 .1318

[14] Yu Q., Wu Z., Chen H.: Dual-function antibacterial surfaces for biomedical applications. Acta Biomateriala, 16, 1-13 (2015). DOI: $10.1016 /$ j.actbio.2015.01.018

[15] Mi L., Jiang S.: Integrated antimicrobial and nonfouling zwitterionic polymers. Angewandte Chemie International Edition, 53, 1746-1754 (2014).

DOI: $10.1002 /$ anie. 201304060

[16] Manikas A. C., Soto Beobide A., Voyiatzis G. A.: Quantitative analysis via surface enhanced Raman Scattering from Ag nano-colloids utilizing an oscillating cell and right-angle collection geometry. Analyst, 134, 587-592 (2009).

DOI: $10.1039 / \mathrm{B} 815053 \mathrm{~B}$

[17] Anastasopoulos J. A., Soto Beobide A., Voyiatzis G. A.: Quantitative surface enhanced Raman scattering measurements at the early stage of active agent release processes. Journal of Raman Spectroscopy, 44, 401405 (2013).

DOI: $10.1002 / j \mathrm{js} .4222$

[18] Bekiari V., Nikolaou K., Koromilas N., Lainioti G., Avramidis P., Hotos G., Kallitsis J. K., Bokias G.: Release of polymeric biocides from synthetic matrices for marine biofouling applications. Agriculture and Agricultural Science Procedia, 4, 445-450 (2015).

DOI: $10.1016 /$ j.aaspro.2015.03.051

[19] Koromilas N. D., Lainioti G. Ch., Gialeli Ch., Barbouri D., Kouravelou K. D., Karamanos N. K., Voyiatzis G. A., Kallitsis J. K.: Preparation and toxicological assessment of functionalized carbon nanotube-polymer hybrids. Plos One, 9, e107029/1-e107029/15 (2014). DOI: $10.1371 /$ journal.pone.0107029

[20] Lee P. C., Meisel D.: Adsorption and surface-enhanced Raman of dyes on silver and gold sols. The Journal of Physical Chemistry, 86, 3391-3395 (1982). DOI: $10.1021 / \mathrm{j} 100214 \mathrm{a} 025$ 
[21] Simomcic B., Tomsic B.: Structures of novel antimicrobial agents for textiles - A review. Textile Research Journal, 80, 1721-1737 (2010).

DOI: $10.1177 / 0040517510363193$

[22] Kougia E., Tselepi M., Vasilopoulos G., Lainioti G. C., Koromilas N. D., Druvari D., Bokias G., Vantarakis A., Kallitsis J. K.: Evaluation of antimicrobial efficiency of new polymers comprised by covalently attached and/or electrostatically bound bacteriostatic species, based on quaternary ammonium compounds. Molecules, 20, 21313-21327 (2015).

DOI: $10.3390 /$ molecules201219768
[23] Dendramis A. L., Schwinn E. W., Sperline R. P.: A surface-enhanced Raman scattering study of CTAB adsorption on copper. Surface Science, 134, 675-688 (1983).

DOI: 10.1016/0039-6028(83)90065-1

[24] Papanu J. S., Hess D. W., Soane D. S., Bell A. T.: Swelling of poly(methyl methacrylate) thin films in low molecular weight alcohols. Journal of Applied Polymer Science, 39, 803-823 (1990).

DOI: $\underline{10.1002 / a p p .1990 .070390404}$ 